\title{
Cinétique de dégradation ruminale des gluten meal
}

\author{
FAV Arruda, MR Alvir, J González, JF Gálvez \\ Universidad Politécnica, Escuela Técnica Superior de Ingenieros Agrónomos, \\ Departamento de Producción Animal, 28040 Madrid, Espagne
}

La dégradation in sacco de la MS et des matières azotées totales (MAT) dans le rumen a été mesurée sur 3 échantillons de gluten meal (GM-1, 2,3) dont les teneurs en amidon et en MAT étaient respectivement de 28,$3 ; 20,5$ et $10,4 \%$, et 61,4 ; 67,2 et $73,5 \%$. L'expérimentation a porté sur 3 moutons munis de canules du rumen, recevant une ration composée de foin et de concentré dans un rapport 2:1, à un niveau de $40 \mathrm{~g} \mathrm{MS} / \mathrm{kg}$ poids ${ }^{0,75}$, distribuée en 2 repas par jour ( 8 et 16 h). Des sachets (pore $46 \mu \mathrm{m}$, dimensions intemes $6,5 \times 10,5 \mathrm{~cm}$ ) contenant $3 \mathrm{~g}$ d'aliment broyé à la grille de $2 \mathrm{~mm}$ ont été placés pendant $2,4,8,16$, $24,36,48$ et $72 \mathrm{~h}$ dans le rumen (sauf $72 \mathrm{~h}$ pour $\mathrm{GM}-1$ ), avec 2 répétitions par aliment et animal.

L'évolution des valeurs de dégradation de la MS et des MAT a montré (fig 1) l'existence de 2 courbes exponentielles consécutives, la deuxième débutant après un temps de séjour pour lequel la première courbe avait déjà pratiquement atteint sa valeur asymptote. Ces 2 courbes ont été ajustées pour chaque animal aux modèles proposés par Ørskov et McDonald (1979) et McDonald (1981), respectivement. Le temps de latence $\left(t_{j}\right)$ correspondant à la dernière courbe a été établi par la résolution mathématique de l'intersection des 2 courbes.

Le calcul de la dégradabilité théorique (DT) en employant seulement la première courbe de dégradation $\left(D T_{1}\right.$ ) (ce qui correspondrait normalement à l'emploi de temps d'incubation non supérieurs à $48 \mathrm{~h})$ conduit à une sous-estimation $(P<0,05$; test tpar couple) de la DT réelle $\left(\mathrm{DT}_{2}\right)$, calculée à partir des 2 courbes (tableau I). L'importance relative de cette sous-estimation varie suivant les échantillons étudiés, de 8,0 à $18,6 \%$ et de 13,3 à $35,3 \%$, pour la MS et les MAT, respectivement.

Ce travail a été financé par la CICYT, Projet GAN89-126.

McDonald I (1981) J Agric Sci Camb 96, 251-252

Ørskov ER, McDonald I (1979) J Agric Sci Camb 92, 499-503

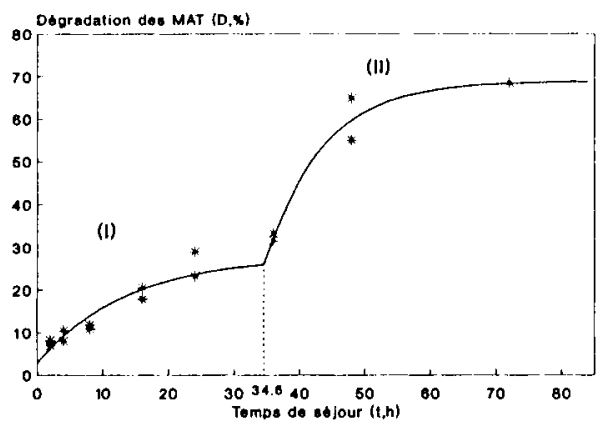

Fig 1. Exemple de modélisation : GM-3, mouton 2. (I) $D=2,84+24,87\left(1-e^{-0,074} \eta\right)$ pour $t<t_{i} ; R^{2}=0,89 ;$ (II) $\mathrm{D}=25,81+43,09$ (1 $\left.e^{-0,116(1-34,6)}\right)$ pour $t>t_{i} ; R^{2}=0,96$.

Tableau I. Temps de latence et dégradabilités théoriques des gluten meal (moyenne \pm écart type).

\begin{tabular}{lcccccc}
\hline & & Matière sèche & \multicolumn{3}{c}{ Matières azotées totales } \\
& GM-1 & GM-2 & GM-3 & GM-1 & GM-2 & GM-3 \\
\hline$t_{i}(\mathrm{~h})$ & $21,0(1,3)$ & $40,0(4,9)$ & $35,8(6,4)$ & $20,8(1,6)$ & $38,7(4,9)$ & $36,3(7,9)$ \\
DT $_{1}(\%)$ & $29,5(0,8) *$ & $33,1(0,9) *$ & $27,5(1,2) *$ & $11,7(1,3) *$ & $20,2(0,3) *$ & $17,9(1,9) *$ \\
DT $_{2}(\%)$ & $35,1(2,8)$ & $36,2(2,7)$ & $33,9(3,5)$ & $18,1(4,4)$ & $23,3(1,6)$ & $24,6(4,5)$ \\
ET $^{* \star}$ & 3,1 & 1,8 & 2,7 & 3,2 & 1,8 & 2,5 \\
\hline
\end{tabular}

$t_{i}$ : temps de latence de la deuxième courbe ; $\mathrm{DT}_{1}, \mathrm{DT}_{2}$ : dégradabilité théorique calculée pour un taux de renouvellement de $4,3 \% \mathrm{~h}^{-1}$ en tenant compte de la première ou des 2 courbes de dégradation, respectivement ; " couple de valeurs significativement différentes $(\rho<0,05)$; "* écart type de la différence entre moyennes. 